\title{
Effect of conjugated estrogens and bazedoxifene on glucose, energy and lipid metabolism in obese postmenopausal women
}

\author{
Kara L Marlatt',*, Dragana Lovre ${ }^{2,3, *}$, Robbie A Beyl ${ }^{1}$, Chandra R Tate ${ }^{2}$, Evelyn K Hayes ${ }^{4}$, Charles F Burant ${ }^{5}$, \\ Eric Ravussin ${ }^{1}$ and Franck Mauvais-Jarvis $\left(\mathbb{D}{ }^{2,3}\right.$
}

${ }^{1}$ Pennington Biomedical Research Center, Baton Rouge, Louisiana, USA, ${ }^{2}$ Tulane University Health Sciences Center, New Orleans, Louisiana, USA, ${ }^{3}$ Southeast Louisiana Veterans Administration Healthcare System, New Orleans, Louisiana, USA, ${ }^{4}$ Baton Rouge General Hospital, Baton Rouge, Louisiana, USA, and ${ }^{5}$ Departments of Internal Medicine, Molecular and Integrative Physiology, University of Michigan, Ann Arbor, Michigan, USA

Correspondence should be addressed to F Mauvais-Jarvis Email

fmauvais@tulane.edu

\begin{abstract}
Objective: Combining conjugated estrogens (CE) with the selective estrogen receptor modulator bazedoxifene (BZA) is a novel, orally administered menopausal therapy. We investigated the effect of CE/BZA on insulin sensitivity, energy metabolism, and serum metabolome in postmenopausal women with obesity.

Design: Randomized, double-blind, crossover pilot trial with washout was conducted at Pennington Biomedical Research Center. Eight postmenopausal women (age 50-60 years, BMI 30-40 kg/m²) were randomized to 8 weeks CE/BZA or placebo. Primary outcome was insulin sensitivity (hyperinsulinemic-euglycemic clamp). Secondary outcomes included body composition (DXA); resting metabolic rate (RMR); substrate oxidation (indirect calorimetry); ectopic lipids ('H-MRS); fat cell size, adipose and skeletal muscle gene expression (biopsies); serum inflammatory markers; and serum metabolome (LC/MS).

Results: CE/BZA treatment produced no detectable effect on insulin sensitivity, body composition, ectopic fat, fat cell size, or substrate oxidation, but resulted in a non-significant increase in RMR (basal: $P=0.06$; high-dose clamp: $P=0.08$ ) compared to placebo. CE/BZA increased serum high-density lipoprotein (HDL)-cholesterol. CE/BZA also increased serum diacylglycerol (DAG) and triacylglycerol (TAG) species containing long-chain saturated, mono- and polyunsaturated fatty acids (FAs) and decreased long-chain acylcarnitines, possibly reflecting increased hepatic de novo FA synthesis and esterification into TAGs for export into very low-density lipoproteins, as well as decreased FA oxidation, respectively $(P<0.05)$. CE/BZA increased serum phosphatidylcholines, phosphatidylethanolamines, ceramides, and sphingomyelins, possibly reflecting the increase in serum lipoproteins $(P<0.05)$. Conclusions: A short treatment of obese postmenopausal women with CE/BZA does not alter insulin action or ectopic fat but increases serum markers of hepatic de novo lipogenesis and TAG production.
\end{abstract}

\section{Introduction}

Menopause is characterized by estrogen deficiency and predisposes women to metabolic disturbances, including visceral adiposity, metabolic syndrome, and type 2 diabetes, especially during the menopausal transition (c) 2020 European Society of Endocrinology Printed in Great Britain
$(1,2,3,4,5)$. Menopausal hormone therapy may delay these metabolic complications (5).

The combination of conjugated estrogens (CE) with the selective estrogen receptor modulator (SERM), 
bazedoxifene (BZA), is approved by the U.S. Food and Drug Administration (FDA) to treat postmenopausal symptoms, including vasomotor symptoms, vulvar-vaginal atrophy, and menopause-induced osteoporosis. CE/BZA is the first estrogen-based, progestin-free, oral menopausal therapy for non-hysterectomized postmenopausal women. Specifically, CE/BZA provides the beneficial effects of CE, while protecting the breast and uterus from estrogenic stimulation with BZA and without the use and side effects of a progestin $(6,7,8,9,10)$.

Using preclinical mouse models of postmenopausal metabolic syndrome, we and others reported that CE/BZA treatment prevents estrogen deficiency-induced obesity, type 2 diabetes, and nonalcoholic fatty liver disease as efficiently as CE treatment alone $(11,12)$. We observed that $\mathrm{CE} / \mathrm{BZA}$ increased fat oxidation and energy expenditure, thereby preventing ectopic lipid accumulation in liver and skeletal muscle and thus enhancing whole-body insulin sensitivity (12). We previously reported that a 12-week treatment of CE/BZA in postmenopausal women with obesity improved fasting $\beta$-cell function and glucose concentrations compared to placebo and without changes in glucose homeostasis during an i.v. glucose tolerance test (13). However, the extent to which CE/BZA improves systemic insulin action and energy expenditure in menopausal women as it does in preclinical models is still unknown. Similar to other orally administered estrogens, $\mathrm{CE} / \mathrm{BZA}$ increases serum triglyceride-containing very lowdensity lipoprotein (VLDL) concentrations $(5,14,15,16)$, yet the mechanism by which this occurs is not understood.

To address these gaps in the literature, we carried out a parallel but separate pilot randomized, double-blind, crossover trial, to investigate the effect of $\mathrm{CE} / \mathrm{BZA}$ in postmenopausal women with obesity - using additional advanced metabolic profiling methods not included in our original study - to assess insulin action (hyperinsulinemiceuglycemic clamp), body composition (dual-energy X-ray absorptiometry; DXA), energy expenditure and substrate oxidation (indirect calorimetry), ectopic lipids (proton magnetic resonance spectroscopy; $\left.{ }^{1} \mathrm{H}-\mathrm{MRS}\right)$, adipose and skeletal muscle gene expression (biopsies), serum inflammatory markers, and serum metabolome (liquid chromatography/mass spectrometry; LC/MS).

\section{Subjects and methods}

\section{Study design}

We conducted a randomized, double-blind, placebocontrolled, crossover pilot trial at Pennington Biomedical
Research Center (PBRC). Participants were recruited, screened, and randomized to receive $0.45 / 20 \mathrm{mg} /$ day of CE/BZA and placebo for an 8-week treatment period, with an 8-week washout between treatments. Participants were instructed to take a single tablet (dose) per day in the morning during each treatment period and return to PBRC to assess adherence and adverse event monitoring at 1,3 , and 5 weeks ( \pm 3 days).

At the end of each 8-week treatment period, participants completed an overnight inpatient stay that included measures of body composition; intrahepatic and skeletal muscle lipid; insulin sensitivity; energy metabolism; fat cell size, adipose tissue and skeletal muscle gene expression; inflammatory markers; and serum and skeletal muscle metabolomics analyses. The study was approved by the PBRC Institutional Review Board and participants provided their written informed consent prior to enrolling in the study. All procedures were conducted in accordance with the 1964 Helsinki declaration and its later amendments or comparable ethical standards.

\section{Study population and eligibility criteria}

Postmenopausal women between 50 and 60 years of age, of all races and ethnicities, and with a BMI between 30 and $40 \mathrm{~kg} / \mathrm{m}^{2}$ were eligible to participate. Postmenopausal status was defined by self-reported absence of menstrual cycles for 1-year and confirmed by a FSH $>30 \mathrm{mIU} / \mathrm{mL}$. Participants had to be within 5 years of their last menstrual period, have had a normal mammogram within the past 12 months, and be cleared to participate by our study physician (EKH). Women could be symptomatic or asymptomatic for menopausal symptoms, such as hot flashes and vaginal dryness. Exclusion criteria included hysterectomy; diabetes mellitus; current or recent use of nicotine or tobacco products (within 3 months); antidiabetic medications, antipsychotics, oral steroids, or recent weight loss; history of major depression; recent use of oral contraception, estrogens, and/or progestogens treatment (within 3 months); and contraindications to estrogen treatment. Other chronic medications were acceptable if taken consistently for 2 months. The CONSORT diagram summarizing throughput is provided in Fig. 1.

\section{Sample size determination}

Our goal in this pilot study was to collect preliminary data to determine the treatment effect and variability estimates to inform the design of a larger clinical trial. 


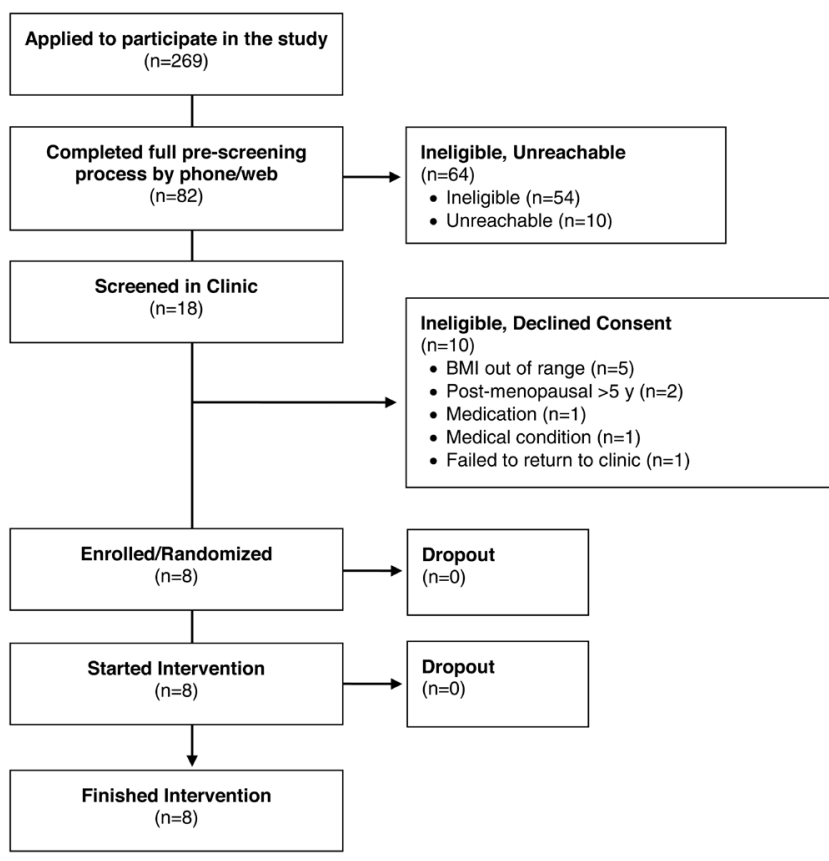

Figure 1

CONSORT Diagram. The CONSORT diagram includes a count of all those subjects screened and enrolled in the present study.

While a formal power analysis was not feasible due to the lack of prior data, we planned to enroll up to ten subjects in order to achieve a goal of eight completed subjects in 1 year with an anticipated dropout rate of $20 \%$. We expected detectable improvements in insulin sensitivity in our sample of completers given the high precision of the hyperinsulinemic-euglycemic clamp technique.

\section{Recruitment and screening strategies}

Participants were continuously recruited via Baton Rouge General Obstetrics and Gynecology Physicians between September 2015 and April 2017 and by the PBRC recruitment core via online advertisements, health promotion events, databases, listserv, and referral sources. During an initial online and telephone screening, participants' age, height, weight and basic medical information were collected. Individuals who did not meet eligibility criteria were excluded. During a screening visit in the morning after an overnight fast, metabolic weight was measured (Scale Tronix 5200, Welch Allyn, Inc.; Skaneateles Falls, NY) while wearing a surgical gown, which was subtracted from total weight. Height, waist and hip circumference, blood pressure, and resting heart rate were also measured.

\section{Study procedures}

Body composition and ectopic fat

Fat mass (FM), fat-free mass (FFM), percent body fat, and bone mineral content (BMC) were measured by dual X-ray absorptiometry whole-body scanner (iDXA; General Electric). Abdominal s.c. adipose tissue (aSAT) mass, visceral adipose tissue (VAT) mass, and total adipose tissue (TAT) mass, as well as intrahepatic lipid (IHL), skeletal muscle intramyocellular lipid (IMCL), and extramyocellular lipid (EMCL) within the soleus and anterior tibialis were all assessed by MRI $/{ }^{1} \mathrm{H}-\mathrm{MRS}$ (3T GE Discovery 750w; Milwaukee, WI). These assessments utilized the Point Resolved Spectroscopy (PRESS) box technique (17). Lipid peaks were normalized to an external oil phantom of known constant concentration (18).

\section{Hyperinsulinemic-euglycemic clamp}

After admission to our inpatient unit, a standard dinner (30\% of the daily energy requirements estimated as Harris-Benedict predicted resting metabolic rate $\mathrm{x}$ activity factor of 1.4) was fed at 19:00 h. Insulin sensitivity was measured early in the morning using a two-step hyperinsulinemic-euglycemic clamp. Insulin was infused for $180 \mathrm{~min}$ at $20 \mathrm{mU} / \mathrm{min} / \mathrm{m}^{2}$ (low-dose) and $120 \mathrm{~min}$ at $120 \mathrm{mU} / \mathrm{min} / \mathrm{m}^{2}$ (high-dose), and variable glucose (20\% dextrose solution) was infused at a rate to maintain plasma glucose concentrations at $90 \mathrm{mg} / \mathrm{dL}$. Endogenous glucose production was determined in the basal state $(0.22$ $\mu \mathrm{mol}\left(6,6-{ }^{2} \mathrm{H}_{2}\right) \mathrm{kg}^{-1} \mathrm{~min}^{-1}$ for $\left.180 \mathrm{~min}\right)$ and during insulinstimulated conditions $\left(1 \%\left(6,6-{ }^{2} \mathrm{H}_{2}\right)\right.$ enrichment of the $20 \%$ dextrose solution). At the low-insulin dose, insulin sensitivity (glucose disposal rate, GDR) was calculated as the sum of the glucose infusion rate plus the residual splanchnic glucose output (SGO) during the final $30 \mathrm{~min}$ (steady-states) of insulin infusion (19). At the high-insulin dose, GDR was equal to glucose insulin infusion rate, since complete endogenous glucose production was achieved. Basal, low-insulin dose, and high-insulin dose steady-state conditions were expressed per $\mathrm{kg}$ of estimated metabolic weight (FFM+17.7 kg) to account for metabolic size (20).

Resting metabolic rate and respiratory quotient were measured by a hood system indirect calorimeter (DeltaTrac; Sensor Medics, Yorba, CA, USA). Blood glucose (DXC 600 Pro; Beckman Coulter), insulin (Immulite 2000 XPi; Siemens), and free fatty acids (FFA) (DXC 600 Pro; Beckman Coulter; reagent kit from Wako) were measured during the three steady-state conditions (baseline, low and high insulin doses). The oxidative component of the GDR 
(or $\mathrm{M}$ value) was calculated as described (21), while the non-oxidative component was calculated as the difference between the total GDR and its oxidative component (22). Urine was collected to determine urinary nitrogen and substrate oxidation.

\section{Clinical chemistry}

Fasting glucose (DXC 600 Pro; Beckman Coulter), estradiol and FSH (Immulite $2000 \mathrm{XPi}$; Siemens), and lipids (DXC 600 Pro; Beckman Coulter) were measured at screening. Markers of inflammation were measured in serum via ELISA, including fibroblast growth factor 21 (FGF21) (23); leptin, plasminogen activator inhibitor-1 (PAI-1), retinol binding protein 4 (RBP4), and Lipocalin 2 (LCN2) (24, 25, 26, 27); adiponectin (28); C-reactive protein (CRP) (29); Gla-type and Glu-type Osteocalcin (Gla-OC and Glu-OC) (30, 31); and Intact Osteocalcin (Intact-OC) (32). Quantitative determination of thiobarbituric acid reactive substances (TBARS) was done using a TBARS assay kit.

\section{Skeletal muscle and adipose tissue Real-Time qPCR}

Skeletal muscle tissue was collected from the vastus lateralis at the basal portion of the clamp using the Bergstrom technique. To extract RNA, tissue was homogenized in $1 \mathrm{~mL}$ TRIzol (Life Technologies) using short bursts of a handheld homogenizer. After $5 \mathrm{~min}$ at room temperature, samples were centrifuged and supernatants transferred to clean tubes. RNA was then extracted using $0.2 \mathrm{~mL}$ chloroform, precipitated with methanol, and then washed with ethanol. RNA concentration and purity were determined using a NanoDrop UV spectrophotometer (BioTek Laboratories) and imaged on a 1\% agarose gel. cDNA was obtained with the Maxima $\mathrm{H}$ Minus master mix with ds DNase kit (Life Technologies). Real-Time PCR was performed using KiCqStart SYBR Green qPCR Ready mix (Sigma) on a CFX96 Touch Real Time System (Bio-Rad). Pre-designed primers used were KiCqStart SYBR Green Primers (Sigma) or PrimePCR ${ }^{\mathrm{TM}}$ SYBR Green Primers (Bio-Rad). Gene expression relative to actin alpha 1, skeletal muscle (ACTA1) was calculated from duplicate samples using the $\Delta \Delta \mathrm{Ct}$ method with CFX Maestro $^{\mathrm{TM}}$ Software (Bio-Rad). Abdominal s.c. adipose tissue was processed similarly to skeletal muscle, except gene expression relative to ribosomal protein S13 (RPS13), which was calculated from duplicate samples using the $\Delta \Delta$ Ct method.

\section{Abdominal fat cell sizing}

Abdominal s.c. adipose tissue (aSAT) was also collected at the basal portion of the clamp using the Bergstrom and Mercedes lipoaspirate techniques. Fat cell size was measured using an osmium tetroxide method adapted from Hirsch and Gallian (33) and Pasarica et al. (34). Cells were counted using the Multisizer 3 Coulter Counter (Beckman Coulter, Miami, FL) at a 400- $\mu \mathrm{m}$ aperture (diameter range: 22-240 $\mu \mathrm{m}$; only cells with a diameter larger than $22 \mu \mathrm{m}$ were included in this analysis), and the average of two runs for approximately 1000-3000 cells each was used to calculate mean fat cell size volume $\left(\mathrm{um}^{3}\right)$. Specifically, mean fat cell size volume is then represented in terms of theoretical spherical diameter. The number of adipose cells above and below the low point (i.e. the nadir) between two cell populations are expressed as percent large fat cells and percent small fat cells, respectively (35). The fat cell ratio (small/large) was also calculated.

\section{Metabolomics}

For global metabolomics analysis, serum or muscle samples were subjected to methanol extraction then split into aliquots for analysis by ultrahigh performance liquid chromatography/mass spectrometry (UHPLC/MS) in the positive (two methods) and negative (two methods) modes as previously described (36). Metabolites were then identified by automated comparison of ion features to a reference library of chemical standards followed by visual inspection for quality control, as previously described (37). For complex lipid analysis, lipids were extracted using methanol:dichloromethane, then analyzed using a SciEx Selexion-5500 QTRAP in positive and negative mode electrospray (MRM mode, >1100 MRMs). Data were derived from integrated area (AUC, area under the curve) of the ion peaks and expressed as the scaled intensity.

\section{Statistical analysis}

All non-omics analyses were performed using SAS version 9.4 software (SAS Institute, Inc.) for the $2 \times 2$ crossover trial design. All tests were performed with a significance level of $\alpha=0.05$. All baseline characteristics are expressed as mean \pm S.D. A linear mixed model was used to assess metabolic differences in the response to CE/BZA and placebo with covariates for time, treatment, and crossover order effects (period and sequence). The model used an unstructured covariance matrix to allow correlation between time points within subjects. Differences in 
treatment outcomes were defined by least squares means \pm s.E.M. from the linear mixed model.

The metabolomics data were analyzed using ArrayStudio (Qiagen, version 7). For the complex lipid panel (CLP), data were generated by first imputing the quantitative $(\mu \mathrm{M})$ data stream, and then rescaled so that the median of each group was equal to 1 . Similarly, for the global metabolomic analysis, data were median-scaled and imputed with the minimum detected quantity (minimum value imputation). Data for either platform were then natural-log transformed prior to statistical analysis using a subject-matched ANOVA model. All metabolomics data are provided with a $P$-value, as well as a respective q-value for an estimate of (adjustment for) the false-discovery rate since multiple comparisons were performed (38). Adjustment for multiple comparisons (q-values) are used when outcomes are considered unrelated. In this case, however, many of these outcomes (metabolites) are interrelated. As a result, we report the q-values for reference, but relied on the $P$-values for interpretation because of the exploratory nature of the metabolomics.

\section{Results}

\section{Baseline characteristics}

At baseline, the enrolled female participants $(n=8 ; 6$ White, 1 Black, and 1 Bi-racial) were $53 \pm 3$ years of age with BMI $35.7 \pm 3.2 \mathrm{~kg} / \mathrm{m}^{2}$. All baseline demographics and laboratory markers are provided in Table 1 . On average, participants had high total cholesterol (240 $\pm 60 \mathrm{mg} /$ $\mathrm{dL})$, high waist circumference $(118.2 \pm 8.1 \mathrm{~cm})$, and borderline high low-density lipoprotein (LDL)-cholesterol (143 $\pm 40 \mathrm{mg} / \mathrm{dL})$. Systolic and diastolic blood pressure, resting heart rate, high-density lipoprotein (HDL)cholesterol, triglycerides, and fasting glucose were within normal limits. The average FSH of $63 \pm 29 \mathrm{mIU} / \mathrm{mL}$ was indicative of the postmenopausal state.

\section{Body composition, ectopic fat, and abdominal fat biology}

All data pertaining to body composition, ectopic fat, and abdominal fat biology are presented in Table 2. Following treatment with CE/BZA, percent body fat was higher compared to placebo, but the ratio of VAT/TAT mass tended to be lower $(P=0.08)$, suggesting that $\mathrm{CE} / \mathrm{BZA}$ produces a redistribution of fat toward s.c. areas. No treatment differences were observed for IHL, soleus IMCL or EMCL, as
Table 1 Baseline characteristics (mean \pm S.D.) of participants $(n=8)$.

Anthropometrics
Age, years
Height, cm
Weight, kg
BMI, kg/m²
Waist circumference, cm
Hip circumference, cm
Waist-to-hip ratio
Systolic blood pressure, mmHg
Diastolic blood pressure, mmHg
Resting heart rate, bpm
Cardiometabolic Risk Factors
Fasting glucose, mg/dL
Total cholesterol, mg/dL
HDL-cholesterol, mg/dL
LDL-cholesterol, mg/dL
Triglycerides, mg/dL
Estradiol, pg/mL
FSH, mlU/mL

$53 \pm 3$

$157.8 \pm 4.0$

$88.9 \pm 7.9$

$35.7 \pm 3.2$

$106.7 \pm 6.7$

$118.2 \pm 8.1$

$0.91 \pm 0.08$

$116 \pm 13$

$74 \pm 11$

$66 \pm 8$

$97 \pm 10$

$240 \pm 60$

$71 \pm 20$

$143 \pm 40$

$133 \pm 44$

$58 \pm 28$

$63 \pm 29$

FSH, follicle-stimulating hormone; HDL, high-density lipoprotein; LDL, low-density lipoprotein.

well as tibialis anterior IMCL or EMCL. No differences were observed in mean fat cell size, the percent of small vs large fat cells, or fat cell ratio (small/large) between treatment groups. As previously reported (39), BMC was higher following treatment with $\mathrm{CE} / \mathrm{BZA}$ compared to placebo.

\section{Insulin sensitivity, markers of insulin resistance, and serum lipids}

All data are presented in Table 3. Basal SGO, low-dose insulin GDR, and high-dose insulin GDR were not different between CE/BZA and placebo groups. Additionally, basal, low-dose, and high-dose insulin measures of glucose, insulin, and FFA concentrations were not different between treatments. CE/BZA treatment did produce higher HDL-cholesterol and a non-significant increase in triglycerides. Total cholesterol and LDL-cholesterol were not different between the treatments.

\section{Energy expenditure and substrate oxidation}

All data related to energy metabolism and substrate utilization are presented in Table 3. Treatment with CE/ BZA tended to result in a higher resting metabolic rate during basal $(P=0.06)$ and high-dose $(P=0.08)$ steadystate conditions. Fat oxidation, as well as carbohydrate oxidation or storage, were not different between treatments during baseline, low-dose insulin, and highdose insulin steady-states. 
Table 2 Effect of CE/BZA on body composition, ectopic fat, and abdominal fat biology. Estimates of means \pm S.D. are listed for all post-treatment outcomes. $P$-values denote treatment differences following adjustment for crossover design (treatment, order of treatment).

\begin{tabular}{|c|c|c|c|}
\hline & \multicolumn{2}{|c|}{ Post-treatment outcomes } & \multirow[b]{2}{*}{ P-value } \\
\hline & Placebo & CE/BZA & \\
\hline \multicolumn{4}{|c|}{$\overline{\text { Body composition (by DXA and MRI) }}$} \\
\hline Weight, kg & $91.3 \pm 6.8$ & $90.6 \pm 7.2$ & 0.24 \\
\hline $\mathrm{BMI}, \mathrm{kg} / \mathrm{m}^{2}$ & $36.6 \pm 2.7$ & $36.4 \pm 2.9$ & 0.26 \\
\hline Percent Body fat, \% & $50.9 \pm 2.5$ & $51.4 \pm 1.9$ & 0.04 \\
\hline Fat mass, kg & $45.5 \pm 4.6$ & $45.7 \pm 4.0$ & 0.71 \\
\hline Fat-free mass, kg & $46.0 \pm 3.5$ & $45.5 \pm 4.0$ & 0.11 \\
\hline $\mathrm{BMC}, \mathrm{g}$ & $2270 \pm 204$ & $2290 \pm 216$ & 0.04 \\
\hline aSAT mass, kg & $12.7 \pm 1.9$ & $12.5 \pm 1.8$ & 0.23 \\
\hline VAT mass, $\mathrm{kg}$ & $2.5 \pm 0.7$ & $2.4 \pm 0.8$ & 0.10 \\
\hline TAT mass, kg & $15.2 \pm 1.9$ & $14.8 \pm 1.8$ & 0.16 \\
\hline VAT/TAT mass & $0.17 \pm 0.05$ & $0.16 \pm 0.05$ & 0.08 \\
\hline \multicolumn{4}{|l|}{ Ectopic fat (by ${ }^{1} \mathrm{H}-\mathrm{MRS}$ ) } \\
\hline $\mathrm{IHL}, \%$ & $9.00 \pm 13.09$ & $8.06 \pm 9.14$ & 0.80 \\
\hline Soleus IMCL, \% & $0.29 \pm 0.27$ & $0.36 \pm 0.47$ & 0.50 \\
\hline Soleus EMCL, \% & $0.32 \pm 0.28$ & $0.43 \pm 0.45$ & 0.60 \\
\hline $\begin{array}{l}\text { Tibialis anterior } \\
\text { IMCL, \% }\end{array}$ & $0.33 \pm 0.23$ & $0.69 \pm 0.50$ & 0.12 \\
\hline $\begin{array}{l}\text { Tibialis anterior } \\
\text { EMCL, \% }\end{array}$ & $1.87 \pm 1.60$ & $4.13 \pm 6.06$ & 0.32 \\
\hline \multicolumn{4}{|c|}{ Abdominal fat cell size (by biopsy) } \\
\hline $\begin{array}{l}\text { Mean fat cell size, } \\
\mu m^{3}\left(\times 10^{6}\right)\end{array}$ & $1.35 \pm 0.4$ & $1.6 \pm 0.4$ & 0.24 \\
\hline $\begin{array}{l}\text { Percent small } \\
\text { fat cells, } \%\end{array}$ & $66.4 \pm 8.7$ & $66.0 \pm 5.5$ & 0.35 \\
\hline $\begin{array}{l}\text { Percent large } \\
\text { fat cells, \% }\end{array}$ & $33.6 \pm 8.7$ & $34.0 \pm 5.5$ & 0.35 \\
\hline $\begin{array}{l}\text { Fat cell ratio } \\
\text { (small/large) }\end{array}$ & $2.19 \pm 1.00$ & $2.00 \pm 0.45$ & 0.42 \\
\hline
\end{tabular}

aSAT, abdominal s.c. adipose tissue; BMC, bone mineral content; CE/BZA, conjugated estrogens/bazedoxifene; DXA, dual-energy X-ray absorptiometry; EMCL, extramyocellular lipid; ${ }^{1} \mathrm{H}-\mathrm{MRS}$, proton magnetic resonance spectroscopy; IHL, intrahepatic lipid; IMCL, intramyocellular lipid; TAT, total adipose tissue; VAT, visceral adipose tissue.

\section{Adipose tissue and skeletal muscle phenotyping and systemic markers of inflammation}

Abdominal adipose tissue and skeletal muscle biopsies were performed to investigate molecular markers of adipose function and insulin action in skeletal muscle. The effects of CE/BZA on adipose tissue and skeletal muscle mRNA expression are presented in Table 4. A higher mRNA expression of adipose tissue-related genes adiponectin $(A D I P O Q)(P=0.02)$ and lipase $\mathrm{E}(L I P E)$ $(P=0.04)$ was observed following CE/BZA treatment compared to placebo. Consistent with GDR results, mRNA expression of skeletal muscle genes involved in mitochondrial function and insulin action was similar between treatment groups. Similarly, no treatment differences were observed for systemic markers of insulin resistance (adiponectin, FGF21, leptin, osteocalcin, Gla-OC, Glu-OC, RBP4), inflammation (CRP, LCN2, PAI-1), or oxidative stress (TBARS) (Table 5).

\section{Metabolomic profiling}

To further assess the effect of CE/BZA on metabolic health, we performed a complete serum metabolomics and lipidomics profiling. The serum metabolome and lipidome comprised a total of 2002 biochemicals, including 1821 compounds of known identity (named biochemicals) and 181 compounds of unknown structural identity (unnamed biochemicals). A complete list of biochemicals with respective $P$-values and q-values is provided in Supplementary Tables 1 and 2 (see section on supplementary materials given at the end of this article).

Treatment with CE/BZA resulted in higher serum diacylglycerols (DAGs) and triacylglycerols (TAGs) composed of long-chain saturated fatty acids (SFA, palmitic C16:0 and arachidic C20:0), monounsaturated FAs (MUFA, palmitoleic C16:1, oleic C18:1 and ecosenoic C20:1), and polyunsaturated FAs (PUFA, linoleic C18:2, arachidonic C20:4, eicosapentaenoic C20:5, and docosahexaenoic $\mathrm{C} 22: 6$ ) compared to placebo (all $P<0.05$ ) (Fig. 2 and Supplementary Table 2). Treatment with CE/ BZA resulted in lower levels of several acylcarnitine species, which are markers of fatty acid (FA) oxidation. These include long-chain SFA (C14, C16 and C18), MUFA (C18:1 and C24:1) and PUFA (C18:2, C20:2 and C20:4) acylcarnitine species (Fig. 2 and Supplementary Table 1). In addition, treatment with $\mathrm{CE} / \mathrm{BZA}$ resulted in higher levels phosphatidylcholines (PCs), phosphatidylinositols (PIs), phosphatidylethanolamines (PEs), sphingomyelins (SMs), and ceramides (CER) (Fig. 3), as well as lower levels of lysophophatidylcholines (LPCs) (Supplementary Table 2). There were no treatment differences in carnitines or ketones (Supplementary Table 1). Additionally, CE/ BZA treatment resulted in lower levels of tyrosine and advanced glycation end products (AGEs) and a higher homoarginine (all $P<0.05$ ) (Supplementary Table 1). Finally, CE/BZA treatment resulted in higher cortisol and thyroxine, possibly reflecting the known effect of orally administered estrogens in increasing liver production of transcortin (Supplementary Table 1), which is not observed during transdermal estradiol therapy (5). No treatment differences in monoacylglycerols (MAGs) or branched-chain amino acids (BCAA) were observed (Supplementary Tables 1 and 2). 
Table 3 Effect of CE/BZA on fasting labs, insulin sensitivity, and metabolism. Estimates of means \pm S.D. are listed for all posttreatment outcomes. $P$-values denote treatment differences following adjustment for crossover design (treatment, order of treatment).

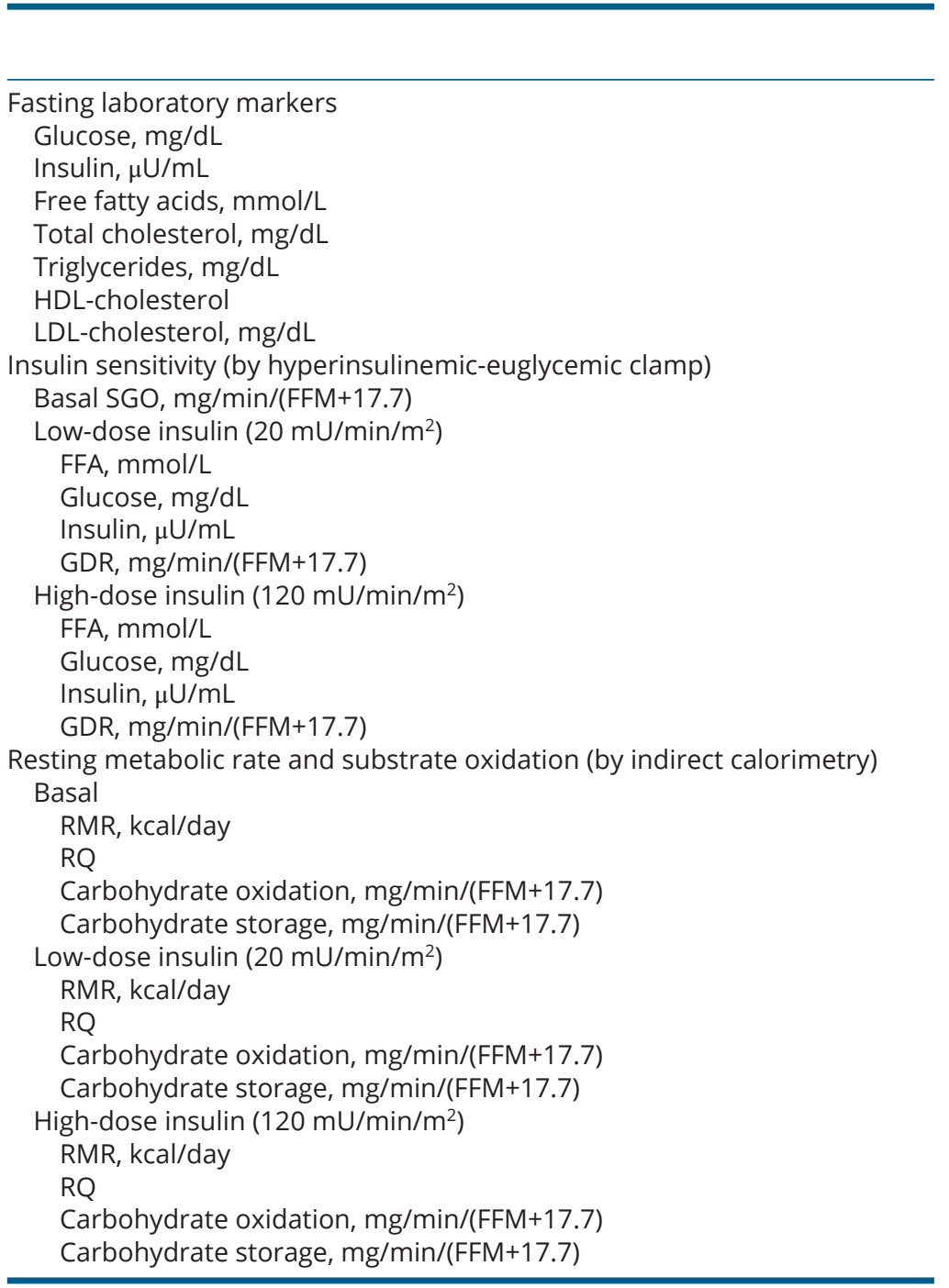

\begin{tabular}{|c|c|c|}
\hline \multicolumn{2}{|c|}{ Post-treatment outcomes } & \multirow[b]{2}{*}{ P-value } \\
\hline Placebo & CE/BZA & \\
\hline $105 \pm 4$ & $102 \pm 4$ & 0.36 \\
\hline $16.6 \pm 2.8$ & $15.7 \pm 2.8$ & 0.53 \\
\hline $0.72 \pm 0.06$ & $0.78 \pm 0.06$ & 0.22 \\
\hline $179 \pm 34$ & $187 \pm 32$ & 0.26 \\
\hline $138 \pm 75$ & $156 \pm 73$ & 0.10 \\
\hline $51 \pm 11$ & $57 \pm 14$ & 0.002 \\
\hline $100 \pm 24$ & $99 \pm 20$ & 0.89 \\
\hline $2.83 \pm 0.41$ & $2.68 \pm 0.32$ & 0.20 \\
\hline $0.13 \pm 0.05$ & $0.13 \pm 0.05$ & 0.74 \\
\hline $90 \pm 1$ & $88 \pm 1$ & 0.16 \\
\hline $37.8 \pm 2.4$ & $34.8 \pm 2.4$ & 0.22 \\
\hline $2.48 \pm 0.91$ & $2.55 \pm 1.04$ & 0.78 \\
\hline ND & ND & - \\
\hline $97 \pm 3$ & $95 \pm 3$ & 0.23 \\
\hline $250.8 \pm 19.3$ & $259.8 \pm 19.3$ & 0.68 \\
\hline $11.99 \pm 4.49$ & $11.68 \pm 4.23$ & 0.66 \\
\hline $1410 \pm 126$ & $1471 \pm 161$ & 0.06 \\
\hline $0.81 \pm 0.03$ & $0.80 \pm 0.03$ & 0.17 \\
\hline $1.2 \pm 0.1$ & $1.0 \pm 0.1$ & 0.27 \\
\hline $1.6 \pm 0.3$ & $1.6 \pm 0.3$ & 0.87 \\
\hline $1379 \pm 94$ & $1386 \pm 137$ & 0.82 \\
\hline $0.85 \pm 0.04$ & $0.83 \pm 0.03$ & 0.19 \\
\hline $1.6 \pm 0.1$ & $1.3 \pm 0.1$ & 0.16 \\
\hline $0.9 \pm 0.3$ & $1.2 \pm 0.3$ & 0.32 \\
\hline $1516 \pm 113$ & $1576 \pm 83$ & 0.08 \\
\hline $0.92 \pm 0.05$ & $0.91 \pm 0.06$ & 0.39 \\
\hline $2.7 \pm 0.3$ & $2.7 \pm 0.3$ & 0.81 \\
\hline $9.3 \pm 1.4$ & $9.0 \pm 1.4$ & 0.75 \\
\hline
\end{tabular}

CE/BZA, conjugated estrogens/bazedoxifene; FFA, free fatty acids; FFM, fat-free mass; GDR, glucose disposal rate; HDL, high-density lipoprotein; LDL, low-density lipoprotein; ND, non-detectable; RMR, resting metabolic rate; RQ, respiratory quotient; SGO, splanchnic glucose output.

By contrast, the skeletal muscle metabolome comprised a total of 652 biochemicals, including 591 named biochemicals and 61 unnamed biochemicals. Unlike in the case of the serum lipidome, no significant differences were observed with regard to DAG, TAG, ACs, PEs, PIs, SMs, or CER between treatment groups. Treatment with CE/BZA decreased two PC species (Supplementary Table 3).

\section{Discuss.ion}

In a crossover pilot trial, we found that treating postmenopausal women with obesity with CE/BZA for 8 weeks did not improve insulin action (primary outcome) or body composition compared to placebo. Similarly, in a randomized controlled trial of postmenopausal women with obesity, we found no effect of a 12-week treatment with CE/BZA on glucose homeostasis using an i.v. glucose tolerance test (13). However, using the homeostatic model assessment to derive $\beta$-cell function (HOMA- $\beta$ ), we observed that women treated with CE/BZA had increased fasting $\beta$-cell function associated with decreased fasting glucose concentrations compared to those treated with placebo (13). In the present trial, we did not observe improvement in HOMA- $\beta$ or glucose levels. Whether differences in study design (parallel arm vs crossover), length of treatment ( 8 vs 12 weeks), or general subject 
Table 4 Effect of CE/BZA on abdominal adipose and skeletal muscle gene expression. Estimates of means \pm S.D. are listed for all post-treatment outcomes. $P$-values denote treatment differences following adjustment for crossover design (treatment, order of treatment).

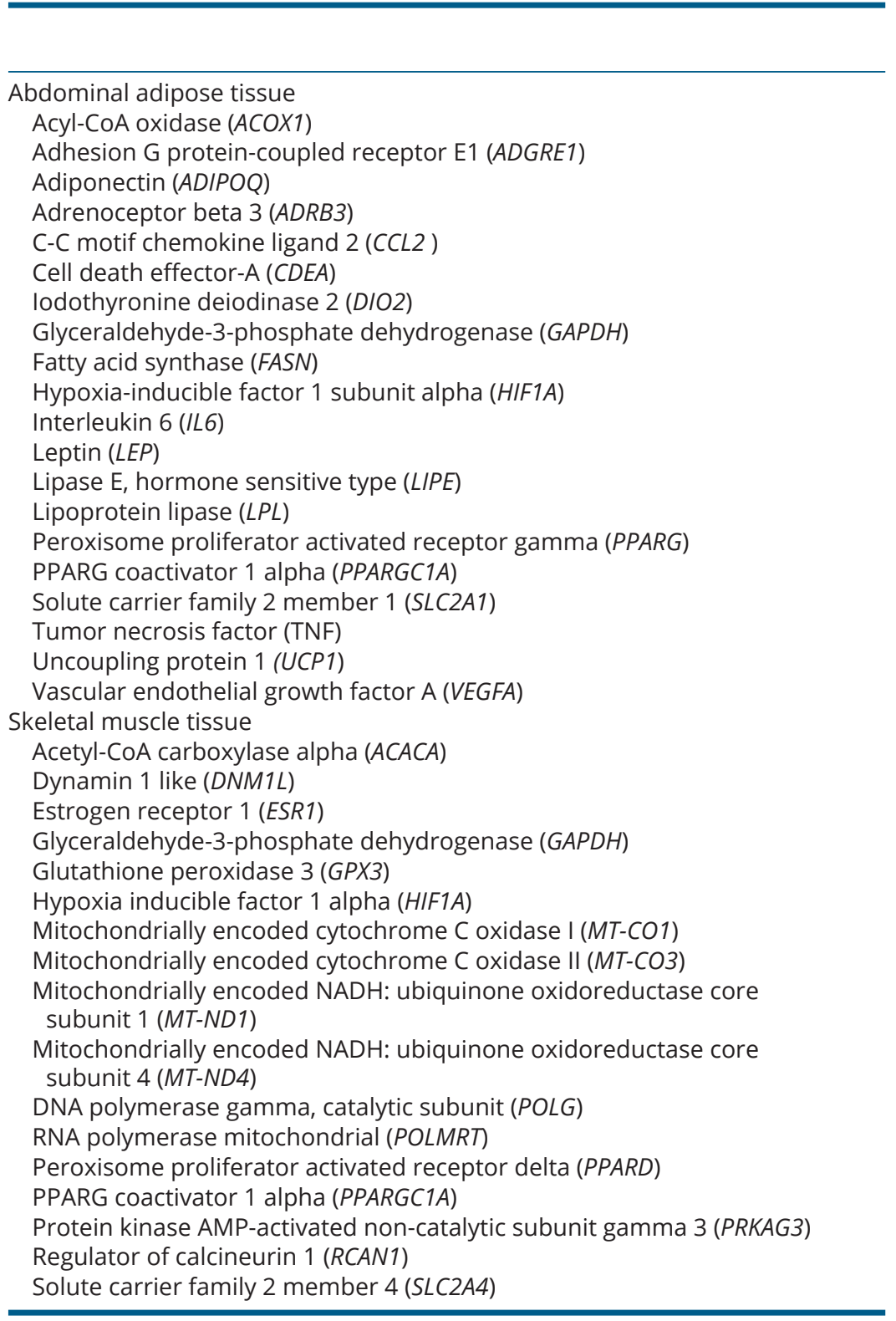

\begin{tabular}{c}
\hline Post-treatmento \\
\hline Placebo \\
\hline \\
$0.82 \pm 0.98$ \\
$0.95 \pm 0.43$ \\
$0.50 \pm 0.22$ \\
$6.59 \pm 10.44$ \\
$1.23 \pm 0.73$ \\
$0.88 \pm 0.31$ \\
$20.07 \pm 23.20$ \\
$0.98 \pm 0.44$ \\
$1.78 \pm 0.90$ \\
$1.47 \pm 2.07$ \\
$2.55 \pm 0.76$ \\
$0.54 \pm 0.41$ \\
$0.47 \pm 0.24$ \\
$0.84 \pm 0.96$ \\
$0.55 \pm 0.42$ \\
$1.94 \pm 2.65$ \\
$0.78 \pm 0.44$ \\
$0.75 \pm 0.74$ \\
$19.02 \pm 20.10$ \\
$0.60 \pm 0.58$ \\
$1.42 \pm 0.44$ \\
$1.20 \pm 0.18$ \\
$1.23 \pm 0.23$ \\
$1.00 \pm 0.17$ \\
$1.39 \pm 0.33$ \\
$1.17 \pm 0.46$ \\
$0.95 \pm 0.42$ \\
$0.69 \pm 0.21$ \\
$0.88 \pm 0.32$ \\
$0.91 \pm 0.38$ \\
$2.70 \pm 3.11$ \\
$0.98 \pm 0.23$ \\
$1.28 \pm 0.19$ \\
$0.97 \pm 0.29$ \\
$1.40 \pm 0.46$ \\
$1.00 \pm 0.23$ \\
$1.42 \pm 0.46$ \\
\end{tabular}

\begin{tabular}{c}
$C E / B Z A$ \\
\hline $1.32 \pm 0.30$ \\
$1.14 \pm 0.23$ \\
$1.22 \pm 0.33$ \\
$2.20 \pm 1.38$ \\
$1.32 \pm 0.26$ \\
$1.61 \pm 1.03$ \\
$1.97 \pm 0.89$ \\
$1.24 \pm 0.30$ \\
$3.46 \pm 2.55$ \\
$1.49 \pm 0.60$ \\
$2.14 \pm 1.41$ \\
$1.22 \pm 0.37$ \\
$0.97 \pm 0.24$ \\
$1.11 \pm 0.33$ \\
$1.19 \pm 0.22$ \\
$1.75 \pm 0.26$ \\
$1.05 \pm 0.18$ \\
$1.15 \pm 1.33$ \\
$2.09 \pm 0.74$ \\
$0.99 \pm 0.23$ \\
$1.26 \pm 0.20$ \\
$1.24 \pm 0.17$ \\
$0.90 \pm 0.15$ \\
$1.19 \pm 0.13$ \\
$0.93 \pm 0.09$ \\
$1.08 \pm 0.43$ \\
$1.17 \pm 0.30$ \\
$1.30 \pm 0.61$ \\
$0.86 \pm 0.15$ \\
$1.00 \pm 0.23$ \\
$1.02 \pm 0.17$ \\
$1.31 \pm 0.57$ \\
$1.08 \pm 0.04$ \\
$0.81 \pm 0.14$ \\
$1.18 \pm 0.31$ \\
$1.07 \pm 0.14$ \\
$1.05 \pm 0.17$ \\
\hline
\end{tabular}

P-value

0.44

0.53

0.02

0.51

0.85

0.22

0.24

0.43

0.27

0.99

0.64

0.07

0.04

0.66

0.07

0.91

0.37

0.63

0.21

0.33

0.60

0.75

0.09

0.17

0.07

0.81

0.49

0.11

0.91

0.73

0.40

0.33

0.14

0.44

0.51

0.68

0.25

CE/BZA, conjugated estrogens/bazedoxifene.

characteristics (body fatness) contribute to the differences in the results we observed between our two studies is unclear.

Because of first-pass liver metabolism, orally administered estrogens (including CE/BZA) alter lipoprotein production, increasing serum HDLcholesterol, decreasing LDL-cholesterol, and increasing TAG-rich very-low-density lipoprotein (VLDL) (5, 14, $15,16)$. However, the mechanisms by which this occurs are not understood. Our results are consistent with these effects of CE/BZA on serum lipids and may provide a mechanistic explanation for the effect of CE/BZA and other orally administered estrogens in increasing serum TAGs. Detailed lipidomics analysis revealed increased serum DAGs and TAGs with no change in these lipids in skeletal muscle, suggesting that these lipids originate from the liver. Most importantly, CE/BZA increases multiple serum DAG and TAG species composed of long-chain SFAs, MUFAs and PUFAs, supporting a role for CE/BZA in stimulating hepatic de novo fatty acid synthesis (producing 
Table 5 Effect of CE/BZA on serum adipokines and systemic inflammation. Estimates of means \pm S.D. are listed for all post-treatment outcome. P-values denote treatment differences following adjustment for crossover design (treatment, order of treatment).

Adiponectin, $\mu \mathrm{g} / \mathrm{mL}$
C-reactive protein (CRP), $\mathrm{mg} / \mathrm{L}$
Fibroblast growth factor 21 (FGF21), $\mathrm{pg} / \mathrm{mL}$
Gla-type osteocalcin (Gla-OC), $\mathrm{ng} / \mathrm{mL}$
Glu-type osteocalcin (Glu-OC), $\mathrm{ng} / \mathrm{mL}$
Glu/Gla ratio
Intact osteocalcin (Intact-OC), $\mathrm{ng} / \mathrm{mL}$
Lipocalin 2 (LCN2), ng/mL
Leptin, ng/mL
Plasminogen activator inhibitor-1 (PAI-1), ng/mL
Retinol-binding protein 4 (RBP4), $\mu \mathrm{mg} / \mathrm{mL}$
Thiobarbituric acid reactive substances (TBARS), nmol/mL

CE/BZA, conjugated estrogens/bazedoxifene.

SFAs), followed by elongation and desaturation of FAs into MUFAs and PUFAs and esterification into DAGs and TAGs for export into VLDL. Indeed, the shortest SFA observed in the $\mathrm{CE} / \mathrm{BZA}$ treatment group is palmitate (C16:0) and rarely $\mathrm{C} 14: 0$, the predominant and final product of de novo lipogenesis. Estrogens are known to increase liver elongase and desaturase activities, the enzymes that catalyze carbon chain extension and remove two hydrogens to create a carbon double bond, respectively. In animal models, estradiol (E2) treatment increases liver desaturase and elongase activity and promotes MUFA and PUFA biosynthesis $(40,41)$. Further, in female mice with humanized lipoprotein fractions via expression of cholesteryl ester transfer protein, E2 treatment promotes hepatic biosynthesis of VLDL and raises plasma VLDLcontaining TAGs (42). Thus, it appears that, in women, CE/BZA raises serum TAGs by increasing hepatic de novo FA synthesis from carbohydrates, incorporation into TAGs and secretion in VLDL.

Consistent with the increased glucose oxidation flux and de novo FA synthesis, we observed a decrease in acylcarnitines containing long-chain SFAs, MUFAs, and PUFAs, which may reflect the inhibition of hepatic FA oxidation. Indeed, FAs are converted to acylcarnitines by carnitine palmitoyltransferase 1 (CPT1) prior to mitochondrial oxidation, and glucose oxidation inhibits CPT1 activity via production of malonyl-CoA (43). A decrease in serum acylcarnitines, thus, reflects the shift toward glucose oxidation and the resulting inhibition of lipid oxidation (44). Short-term oral estrogens treatments are known to decrease lipid oxidation $(45,46)$. Surprisingly, we observe no change in carbohydrate oxidation during clamp conditions or indirect calorimetry, which we

\begin{tabular}{|c|c|c|}
\hline \multicolumn{2}{|c|}{ Post-treatment outcomes } & \multirow[b]{2}{*}{$P$-value } \\
\hline Placebo & CE/BZA & \\
\hline $7.63 \pm 2.81$ & $7.81 \pm 2.17$ & 0.62 \\
\hline $2.04 \pm 0.89$ & $2.58 \pm 1.27$ & 0.33 \\
\hline $391.44 \pm 348.46$ & $317.88 \pm 156.98$ & 0.53 \\
\hline $7.91 \pm 2.64$ & $8.07 \pm 2.81$ & 0.87 \\
\hline $4.87 \pm 1.94$ & $6.28 \pm 3.63$ & 0.28 \\
\hline $0.64 \pm 0.24$ & $0.81 \pm 0.41$ & 0.30 \\
\hline $4.97 \pm 2.13$ & $5.13 \pm 1.87$ & 0.88 \\
\hline $13.31 \pm 13.56$ & $10.76 \pm 9.57$ & 0.24 \\
\hline $50.00 \pm 8.93$ & $50.44 \pm 11.71$ & 0.91 \\
\hline $23.17 \pm 10.48$ & $23.63 \pm 10.60$ & 0.88 \\
\hline $23.42 \pm 11.80$ & $23.96 \pm 12.04$ & 0.81 \\
\hline $7.65 \pm 2.62$ & $6.92 \pm 2.01$ & 0.45 \\
\hline
\end{tabular}

interpret as the ultra-sensitivity of mass spectrometry to detect changes in metabolites and metabolic fluxes long before physiological or clinical changes can be observed using classical physiological methods (47).

The most abundant lipids in VLDL are in descending order, that is, PE, CER, PC, and SM (48). Therefore, the increase in these lipid species following CE/BZA treatment possibly reflect the estrogens-induced synthesis and export of TAG-containing VLDL.

Taken together, our lipidomic analysis along with published literature allow us to propose a model in which CE/BZA (and likely all orally administered estrogens) increases hepatic de novo lipogenesis, esterification of SFA-, MUFA-, and PUFA-containing TAGs and stimulates the assembly into VLDL for secretion, which raises circulating VLDL-TAGs. Although CE/BZA treatment inhibits FA oxidation, it did not result in hepatic lipid accumulation as measured by MRS. Thus, CE/BZA exports FA into VLDLTAG, which prevents hepatic TAG accumulation and steatosis. Our proposed mechanism of CE/BZA action on liver lipogenesis aforementioned is summarized in Fig. 4.

Unlike in the present study, using female mice, we previously observed that orally administered CE/ BZA promotes lipid oxidation and increases energy expenditure, which prevents estrogen-deficiency-induced visceral adiposity, ectopic lipid accumulation, systemic inflammation and insulin resistance (12). This highlights the difference between rodent and human investigations and the limited translational potential of some rodent studies.

Our study has limitations. The goal of a pilot study is not to be powered to detect differences in outcomes and no formal power calculations was performed for the present 
A

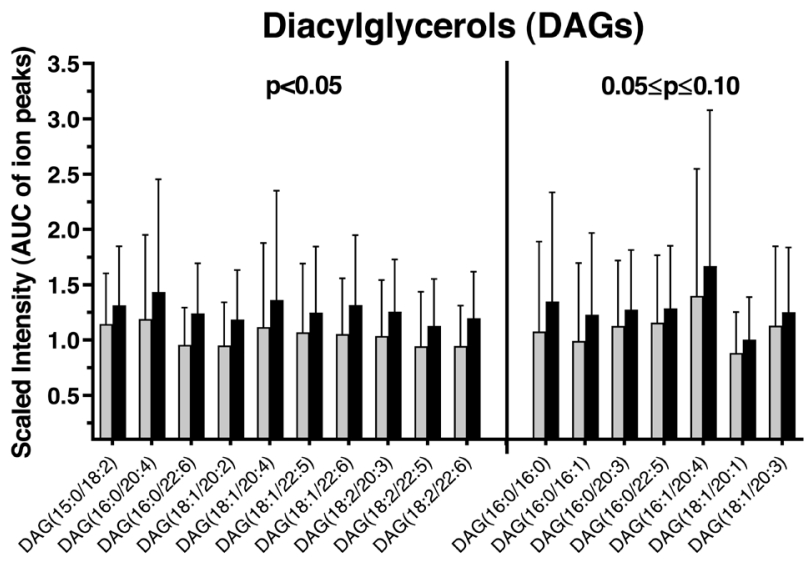

Placebo

CE/BZA

$\mathrm{B}$
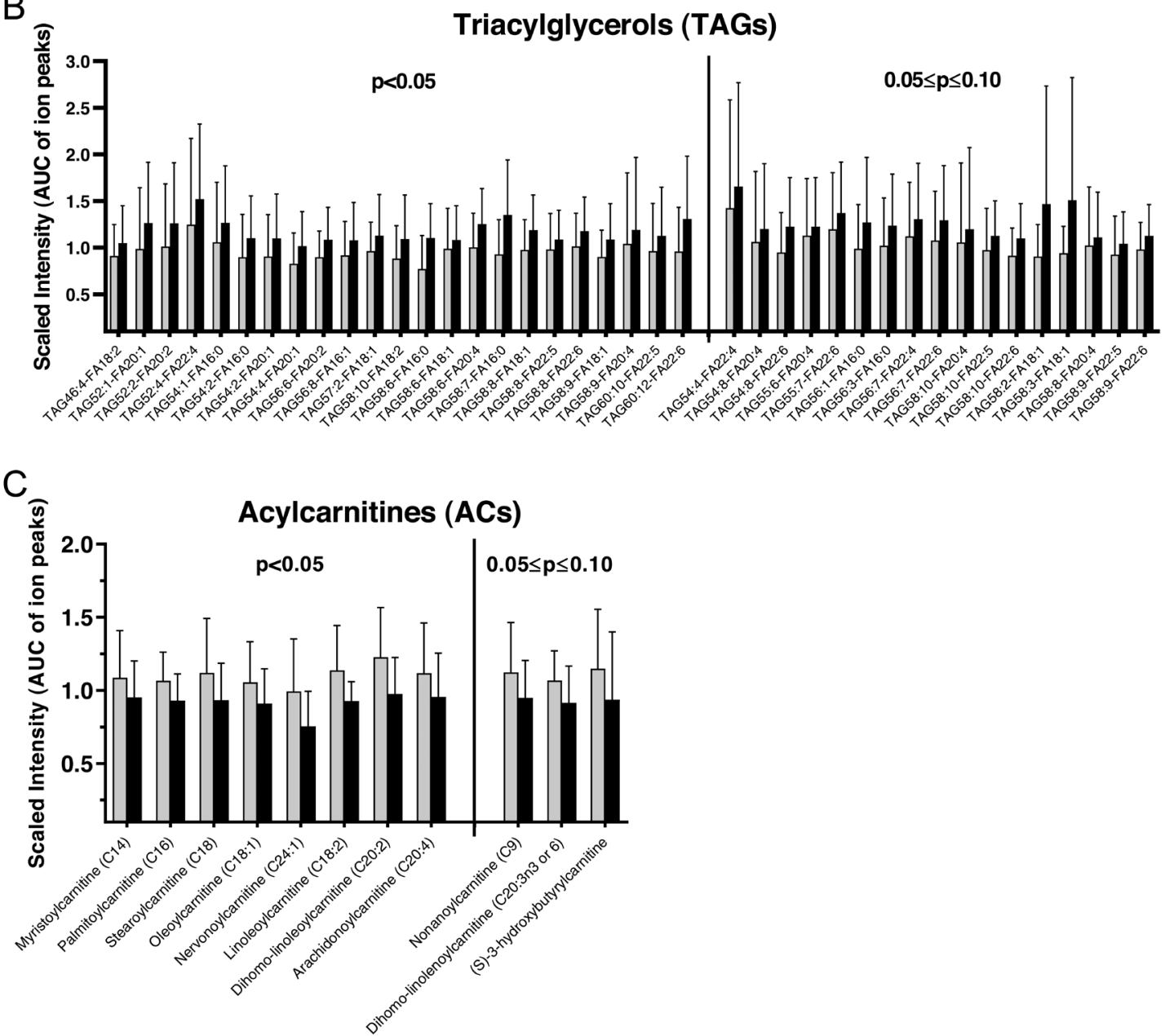

Figure 2

Effect of CE/BZA on serum markers of FA synthesis and oxidation. Metabolites that achieved statistical significance included diacylglycerols (DAGs; Panel A), triacylglycerols (TAGs; Panel B), and acylcarnitines (ACs; Panel C). Median-scaled, imputed data (see methods for more details) are expressed as mean \pm S.D. CE/BZA treatment is indicated by filled black bars, while placebo treatment is indicated by filled grey bars. Lipid species that demonstrated significant (left; $P<0.05$ ) differences between CE/BZA and placebo treatment are noted, as well as those lipid species that trended towards a significant difference (right; $0.05 \leq P \leq$ 0.10). Data are shown as scaled intensity or the area under the curve (AUC) of the ion peaks. 

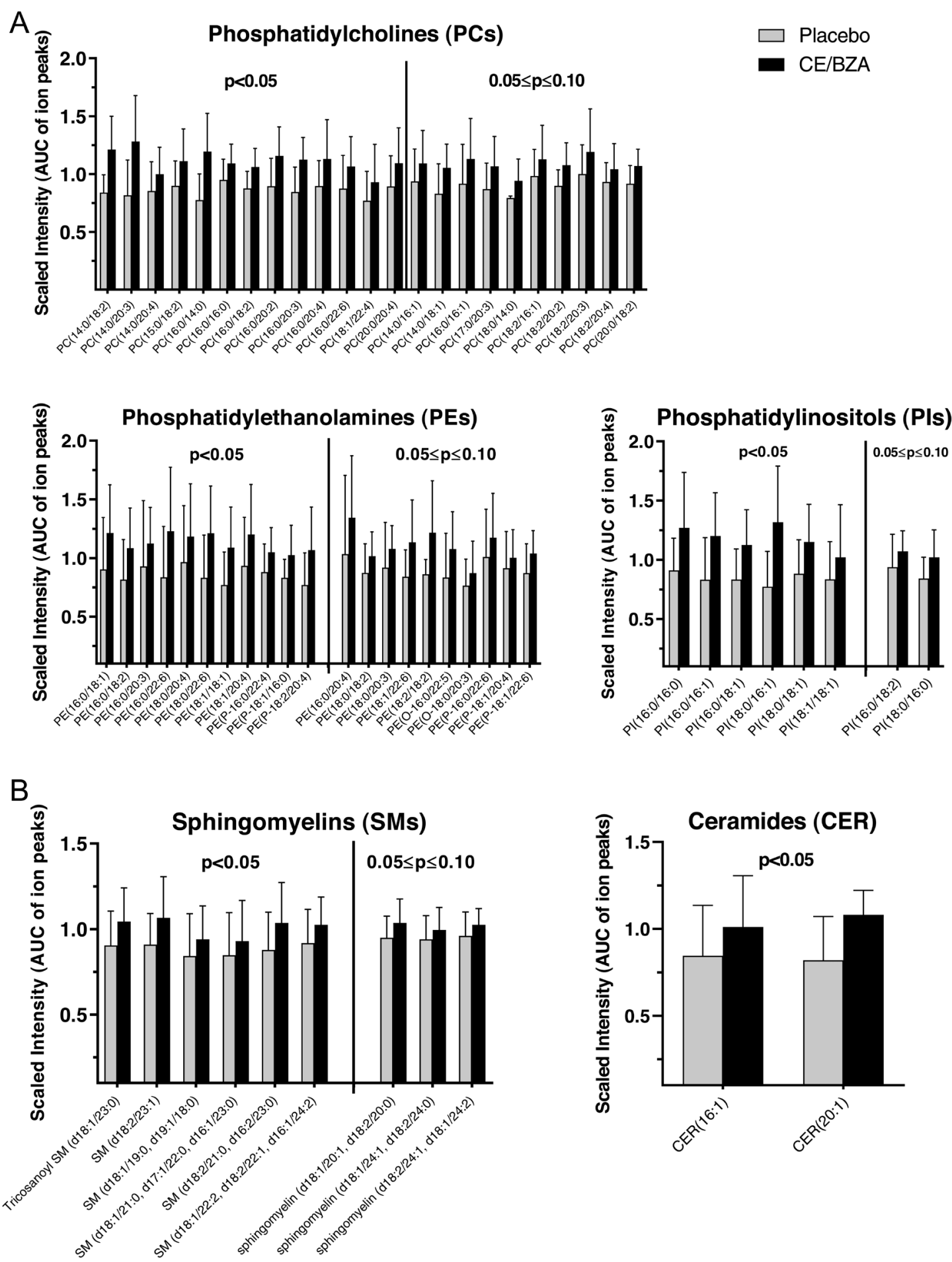

Figure 3

Effect of CE/BZA on serum phospholipids. Metabolites that achieved statistical significance included phosphatidylcholines (PCs), phosphatidylethanolamines (PEs), and phosphatidylinositols (PIs) (Panel A), as well as sphingomyelins (SMs) and ceramides (CER) (Panel B), are expressed as mean \pm S.D. CE/BZA treatment is indicated by filled black bars, while placebo treatment is indicated by filled grey bars. Lipid species that demonstrated significant (left; $P<0.05$ ) differences between CE/BZA and placebo treatment are noted, as well as those lipid species that trended towards a significant difference (right; $0.05 \leq P \leq 0.10$ ). Data are shown as scaled intensity or the area under the curve (AUC) of the ion peaks. 


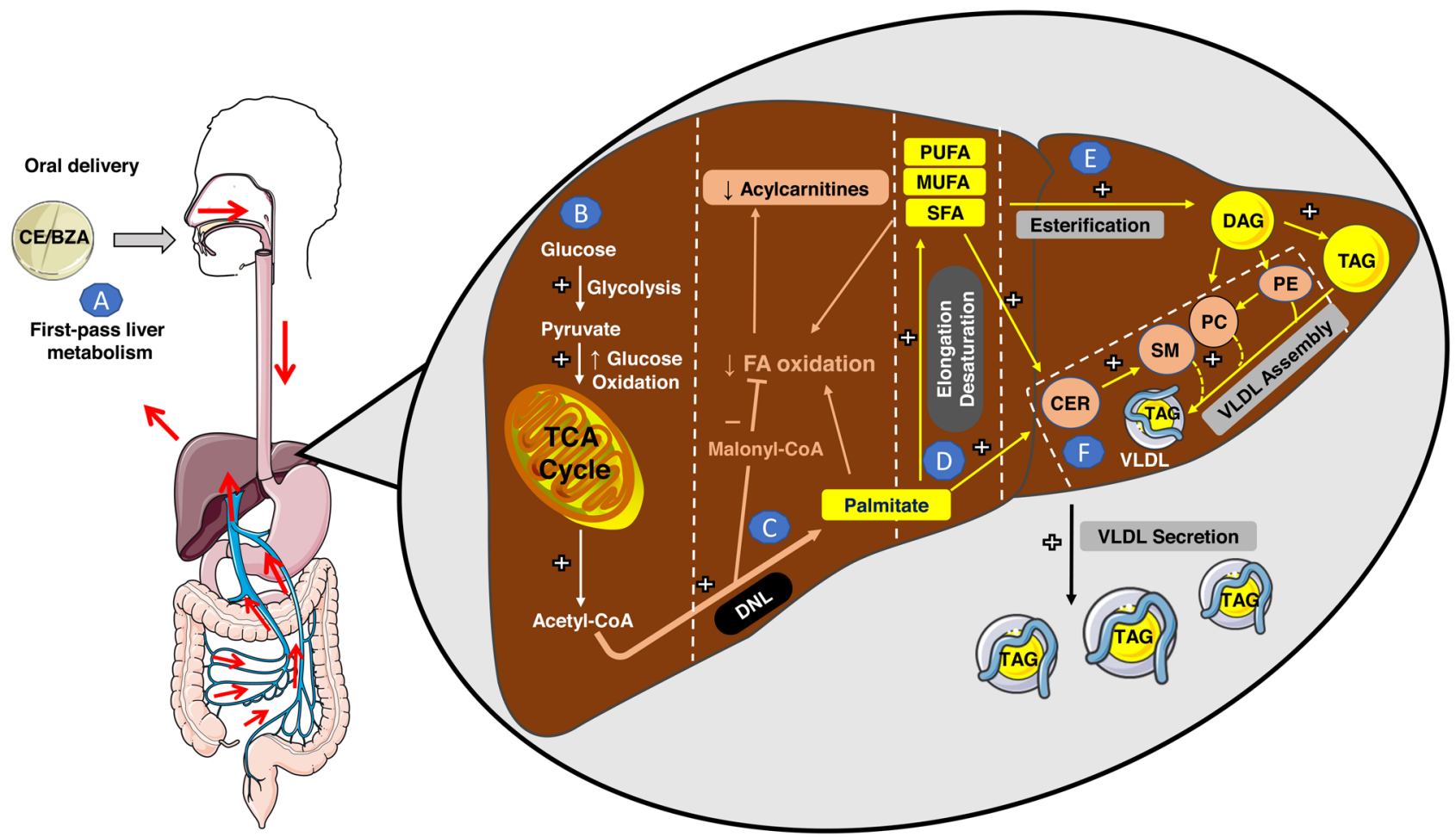

\section{Figure 4}

Proposed mechanism for CE/BZA stimulation of hepatic TAG secretion. (A) Due to first-pass liver metabolism, orally administered CE/BZA increases liver estrogens exposure; (B) CE/BZA increases hepatocyte glucose oxidation in the TCA cycle to produce Acetyl-CoA; (C) Acetyl-CoA is used for de novo lipogenesis (DNL), which produces palmitate (C16:0). Simultaneously, the DNL intermediate malonyl-CoA inhibits mitochondrial FA oxidation, which decreases production of acylcarnitines; (D) Palmitate undergoes elongation and desaturation to produce SFAs, MUFAs, and PUFAs; (E) SFAs, MUFAs, and PUFAs are then esterified into DAGs and TAGs; and (F) the increased production of palmitate, SFAs, and DAGs induce the production of SMs and CERs, as well as PEs and PCS, which will be incorporated with TAGs in the assembly of VLDL to increase hepatic TAG-containing VLDL secretion. CE, conjugated estrogens; BZA, bazedoxifene; DNL, de novo lipogenesis; FA, fatty acid; SFA, saturated fatty acids; MUFA, monounsaturated fatty acids; PUFA, polyunsaturated fatty acids; DAGs, diacylglycerols; TAGs, triacylglycerols; SMs, sphingomyelins; CERs, ceramides; PEs, phosphatidylethanolamines; PCs, phosphatidylcholines; and VLDL, very-low-density lipoprotein.

pilot trial. This may explain why many of our findings are non-significant. These preliminary findings require a larger, fully powered study to be validated. However, these data can now be used to inform the design, adequately power and estimate sample size of future clinical trials that will examine the effect of CE/BZA treatment on similar outcomes. We were also unable to perform pre-intervention assessment of metabolic function and relied on outcome differences at the end of each 8-week treatment period. Furthermore, the shorter 8-week treatment duration may have been insufficient to observe improvements in metabolic function using classical physiological testing. Future randomized, parallel arm studies should include larger sample sizes with pre-intervention and postintervention assessments over a longer period of time.
In conclusion, treatment of obese postmenopausal women with CE/BZA increases serum markers of hepatic de novo lipogenesis and TAG production without changes in insulin action or ectopic fat.

\section{Supplementary materials}

This is linked to the online version of the paper at https://doi.org/10.1530/ EJE-20-0619.

\section{Declaration of interest}

F M J was a scientific advisory board member and received investigatorinitiated research funding from Pfizer, Inc. The other authors have no conflicts of interest to report. 


\section{Funding}

This work is supported, in part, by the National Institute of General Medical Sciences of the National Institutes of Health, which funds the Louisiana Clinical and Translational Science (LA CaTS) Center (U54-GM104940), and NORC Center Grants (P30-DK072476 and P30-DK089503). K L M was supported by the NIDDK sponsored Ruth L. Kirschstein National Research Service T32 Research Training Grant (T32-DK064584). D L was supported by Eunice Kennedy Shriver National Institute of Child Health \& Human Development of the Building Interdisciplinary Research Careers in Women's Health (BIRCWH) Scholars Program (K12-HD043451) and U54-GM104940 from the National Institute of General Medical Sciences, which funds the Louisiana Clinical and Translational Science Center. F M J was supported by NIH grants (R01-DK074970, R01-DK107444) and a Department of Veterans Affairs Merit Review Award (\#BX003725).

\section{Data availability}

The datasets generated during and/or analyzed during the current study are not publicly available but are available from the corresponding author on reasonable request.

\section{References}

1 Keller C, Larkey L, Distefano JK, Boehm-Smith E, Records K, Robillard A, Veres S, Al-Zadjali M \& O'Brian AM. Perimenopausal obesity. Journal of Women's Health 201019 987-996. (https://doi. org/10.1089/jwh.2009.1547)

2 Lemay A, Turcot L, Déchêne F, Dodin S \& Forest JC. Hyperinsulinemia in nonobese women reporting a moderate weight gain at the beginning of menopause: a useful early measure of susceptibility to insulin resistance. Menopause 201017 321-325. (https://doi.org/10.1097/gme.0b013e3181b7c521)

3 Lovejoy JC, Champagne CM, Smith SR, De Jonge L \& Xie H. Ethnic differences in dietary intakes, physical activity, and energy expenditure in middle-aged, premenopausal women: the Healthy Transitions Study. American Journal of Clinical Nutrition $2001 \mathbf{7 4}$ 90-95. (https://doi.org/10.1093/ajcn/74.1.90)

4 Lovejoy JC, Champagne CM, De Jonge L, Xie H \& Smith SR. Increased visceral fat and decreased energy expenditure during the menopausal transition. International Journal of Obesity 200832 949-958. (https://doi.org/10.1038/ijo.2008.25)

5 Mauvais-Jarvis F, Manson JE, Stevenson JC \& Fonseca VA. Menopausal hormone therapy and type 2 diabetes prevention: evidence, mechanisms, and clinical implications. Endocrine Reviews 201738 173-188. (https://doi.org/10.1210/er.2016-1146)

6 Santen RJ, Kagan R, Altomare CJ, Komm B, Mirkin S \& Taylor HS. Current and evolving approaches to individualizing estrogen receptor-based therapy for menopausal women. Journal of Clinical Endocrinology and Metabolism 201499 733-747. (https://doi. org/10.1210/jc.2013-3680)

7 Komm BS \& Mirkin S. Evolution of the tissue selective estrogen complex (TSEC). Journal of Cellular Physiology 2013228 1423-1427. (https://doi.org/10.1002/jcp.24324)

8 Pinkerton JV, Harvey JA, Pan K, Thompson JR, Ryan KA, Chines AA $\&$ Mirkin S. Breast effects of bazedoxifene-conjugated estrogens: a randomized controlled trial. Obstetrics and Gynecology 2013121 959-968. (https://doi.org/10.1097/AOG.0b013e31828c5974)

9 Komm BS. A new approach to menopausal therapy: the tissue selective estrogen complex. Reproductive Sciences 200815 984-992. (https://doi.org/10.1177/1933719108325759)

10 Kharode Y, Bodine PVN, Miller CP, Lyttle CR \& Komm BS. The pairing of a selective estrogen receptor modulator, bazedoxifene, with conjugated estrogens as a new paradigm for the treatment of menopausal symptoms and osteoporosis prevention. Endocrinology 2008149 6084-6091. (https://doi.org/10.1210/en.2008-0817)

11 Barrera J, Chambliss KL, Ahmed M, Tanigaki K, Thompson B, McDonald JG, Mineo C \& Shaul PW. Bazedoxifene and conjugated estrogen prevent diet-induced obesity, hepatic steatosis, and type 2 diabetes in mice without impacting the reproductive tract. American Journal of Physiology: Endocrinology and Metabolism 2014307 E345-E354. (https://doi.org/10.1152/ajpendo.00653.2013)

12 Kim JH, Meyers MS, Khuder SS, Abdallah SL, Muturi HT, Russo L, Tate CR, Hevener AL, Najjar SM, Leloup C et al. Tissue-selective estrogen complexes with Bazedoxifene prevent metabolic dysfunction in female mice. Molecular Metabolism 20143 177-190. (https://doi.org/10.1016/j.molmet.2013.12.009)

13 Lovre D, Peacock E, Katalenich B, Moreau C, Xu B, Tate C, Utzschneider KM, Gautier JF, Fonseca V \& Mauvais-Jarvis F. Conjugated estrogens and bazedoxifene improve $\beta$ cell function in obese menopausal women. Journal of the Endocrine Society 20193 1583-1594. (https://doi.org/10.1210/js.2019-00074)

14 Stevenson JC, Chines A, Pan K, Ryan KA \& Mirkin S. A pooled analysis of the effects of conjugated estrogens/bazedoxifene on lipid parameters in postmenopausal women from the Selective estrogens, Menopause, and Response to Therapy (SMART) trials. Journal of Clinical Endocrinology and Metabolism 2015100 2329-2338. (https:// doi.org/10.1210/jc.2014-2649)

15 Walsh BW, Schiff I, Rosner B, Greenberg L, Ravnikar V \& Sacks FM. Effects of postmenopausal estrogen replacement on the concentrations and metabolism of plasma lipoproteins. New England Journal of Medicine 1991325 1196-1204. (https://doi.org/10.1056/ NEJM199110243251702)

16 Walsh BW \& Sacks FM. Effects of low dose oral contraceptives on very low density and low density lipoprotein metabolism. Journal of Clinical Investigation 199391 2126-2132. (https://doi.org/10.1172/ JCI116437)

17 Larson-Meyer DE, Newcomer BR \& Hunter GR. Influence of endurance running and recovery diet on intramyocellular lipid content in women: a 1H NMR study. American Journal of Physiology: Endocrinology and Metabolism 2017282 E95-E106. (https://doi. org/10.1152/ajpendo.2002.282.1.E95)

18 Perseghin G, Scifo P, De Cobelli F, Pagliato E, Battezzati A, Arcelloni C, Vanzulli A, Testolin G, Pozza G, Del Maschio A et al. Intramyocellular triglyceride content is a determinant of in vivo insulin resistance in humans: a $1 \mathrm{H}-13 \mathrm{C}$ nuclear magnetic resonance spectroscopy assessment in offspring of type 2 diabetic parents. Diabetes $1999 \mathbf{4 8}$ 1600-1606. (https://doi.org/10.2337/diabetes.48.8.1600)

19 DeFronzo RA, Tobin JD \& Andres R. Glucose clamp technique: a method for quantifying insulin secretion and resistance. American Journal of Physiology 1979237 E214-E223. (https://doi.org/10.1152/ ajpendo.1979.237.3.E214)

20 Lillioja S \& Bogardus C. Obesity and insulin resistance: lessons learned from the Pima Indians. Diabetes/Metabolism Reviews 19884 517-540. (https://doi.org/10.1002/dmr.5610040508)

21 Jéquier E, Acheson K \& Schutz Y. Assessment of energy expenditure and fuel utilization in man. Annual Review of Nutrition 19877 187-208. (https://doi.org/10.1146/annurev.nu.07.070187.001155)

22 Ravussin E, Bogardus C, Schwartz RS, Robbins DC, Wolfe RR, Horton ES, Danforth Jr E \& Sims EA. Thermic effect of infused glucose and insulin in man. Decreased response with increased insulin resistance in obesity and noninsulin-dependent diabetes mellitus. Journal of Clinical Investigation 198372 893-902. (https:// doi.org/10.1172/JCI111060)

23 RRID:AB_2783729. Human FGF-21. (available at: https://scicrunch. org/resolver/RRID:AB_2783729)

24 RRID:AB_2800328. Human leptin. (available at: https://scicrunch. org/resolver/RRID:AB_2800328)

25 RRID:AB_2800329. Human PAI-1. (available at: https://scicrunch. org/resolver/RRID:AB_2800329) 
26 RRID:AB_2800330. Human RBP4. (available at: https://scicrunch. org/resolver/RRID:AB_2800330)

27 RRID:AB_2800331. Human Lipocalin-2/NGAL. (available at: https:// scicrunch.org/resolver/RRID:AB_2800331)

28 RRID:AB_2800326. Adiponectin. (available at: https://scicrunch.org/ resolver/RRID:AB_2800326)

29 RRID:AB_2575140. Human CRP. (available at: https://scicrunch.org/ resolver/RRID:AB_2575140)

30 RRID:AB_2800333. Human Gla-type osteocalcin. (available at: https://scicrunch.org/resolver/RRID:AB_2800333)

31 RRID:AB_2800334. Human undercarboxylated osteocalcin (Glu-OC). [Internet]. (available at: https://scicrunch.org/resolver/ RRID:AB_2800334)

32 RRID:AB_2800325. Intact osteocalcin. (available at: https:// scicrunch.org/resolver/RRID:AB_2800325)

33 Hirsch J \& Gallian E. Methods for the determination of adipose cell size in man and animals. Journal of Lipid Research 19689 110-119. (available at: http://www.ncbi.nlm.nih.gov/pubmed/4295346)

34 Pasarica M, Xie H, Hymel D, Bray G, Greenway F, Ravussin E \& Smith SR. Lower total adipocyte number but no evidence for small adipocyte depletion in patients with type 2 diabetes. Diabetes Care 200932 900-902. (https://doi.org/10.2337/dc08-2240)

35 McLaughlin T, Sherman A, Tsao P, Gonzalez O, Yee G, Lamendola C, Reaven GM \& Cushman SW. Enhanced proportion of small adipose cells in insulin-resistant vs insulin-sensitive obese individuals implicates impaired adipogenesis. Diabetologia 200750 1707-1715. (https://doi.org/10.1007/s00125-007-0708-y)

36 Long T, Hicks M, Yu HC, Biggs WH, Kirkness EF, Menni C, Zierer J, Small KS, Mangino M, Messier $\mathrm{H}$ et al. Whole-genome sequencing identifies common-to-rare variants associated with human blood metabolites. Nature Genetics 201749 568-578. (https://doi. org/10.1038/ng.3809)

37 Dehaven CD, Evans AM, Dai H \& Lawton KA. Organization of GC/ MS and LC/MS metabolomics data into chemical libraries. Journal of Cheminformatics 20102 9. (https://doi.org/10.1186/1758-2946-2-9)

38 Storey JD \& Tibshirani R. Statistical significance for genomewide studies. PNAS 2003100 9440-9445. (https://doi.org/10.1073/pnas.1530509100)

39 Christopher Gallagher JC, Palacios S, Ryan KA, Yu CR, Pan K, Kendler DL, Mirkin S \& Komm BS. Effect of conjugated estrogens/ bazedoxifene on postmenopausal bone loss: pooled analysis of two randomized trials. Menopause 201623 1083-1091. (https://doi. org/10.1097/GME.0000000000000694)
40 Marks KA, Kitson AP, Shaw B, Mutch DM \& Stark KD. StearoylCoA desaturase 1, elongase 6 and their fatty acid products and precursors are altered in ovariectomized rats with $17 \beta$-estradiol and progesterone treatment. Prostaglandins, Leukotrienes, and Essential Fatty Acids 201389 89-96. (https://doi.org/10.1016/j. plefa.2013.05.002)

41 Zhang M, Li CC, Li F, Li H, Liu XJ, Loor JJ, Kang XT \& Sun GR. Estrogen promotes hepatic synthesis of long-chain polyunsaturated fatty acids by regulating ELOVL5 at post-transcriptional level in laying hens. International Journal of Molecular Sciences 2017181405. (https://doi.org/10.3390/ijms18071405)

42 Palmisano BT, Le TD, Zhu L, Lee YK \& Stafford JM. Cholesteryl ester transfer protein alters liver and plasma triglyceride metabolism through two liver networks in female mice. Journal of Lipid Research 201657 1541-1551. (https://doi.org/10.1194/jlr. M069013)

43 Mcgarry JD, Mannaerts GP \& Foster DW. A possible role for malonylCOA in the regulation of hepatic fatty acid oxidation and ketogenesis. Journal of Clinical Investigation 197760 265-270. (https://doi. org/10.1172/JCI108764)

44 Ramos-Roman MA, Sweetman L, Valdez MJ \& Parks EJ. Postprandial changes in plasma acylcarnitine concentrations as markers of fatty acid flux in overweight and obesity. Metabolism: Clinical and Experimental 201261 202-212. (https://doi.org/10.1016/j. metabol.2011.06.008)

45 Lwin R, Darnell B, Oster R, Lawrence J, Foster J, Azziz R \& Gower BA. Effect of oral estrogen on substrate utilization in postmenopausal women. Fertility and Sterility 200890 1275-1278. (https://doi. org/10.1016/j.fertnstert.2007.07.1317)

46 Gormsen LC, Høst C, Hjerrild BE, Pedersen SB, Nielsen S, Christiansen JS \& Gravholt CH. Estradiol acutely inhibits whole body lipid oxidation and attenuates lipolysis in subcutaneous adipose tissue: a randomized, placebo-controlled study in postmenopausal women. European Journal of Endocrinology 2012167 543-551. (https:// doi.org/10.1530/EJE-12-0422)

47 Newgard CB. Metabolomics and metabolic diseases: where do we stand? Cell Metabolism 201725 43-56. (https://doi.org/10.1016/j. cmet.2016.09.018)

48 Wiesner P, Leidl K, Boettcher A, Schmitz G \& Liebisch G. Lipid profiling of FPLC-separated lipoprotein fractions by electrospray ionization tandem mass spectrometry. Journal of Lipid Research 2009 50 574-585. (https://doi.org/10.1194/jlr.D800028-JLR200)

Received 3 June 2020

Revised version received 24 June 2020

Accepted 2 July 2020 